\title{
Electric-field-controlled conductance of "metallic" polymers in a transistor structure
}

\author{
Fang-Chi Hsu, ${ }^{1}$ Vladimir N. Prigodin, ${ }^{1}$ and Arthur J. Epstein ${ }^{1,2}$ \\ ${ }^{1}$ Department of Physics, The Ohio State University, Columbus, Ohio 43210-1117, USA \\ ${ }^{2}$ Department of Chemistry, The Ohio State University, Columbus, Ohio 43210-1185, USA
}

(Received 30 June 2006; published 28 December 2006)

\begin{abstract}
It was recently reported that use of a doped "metallic" polymer as the active channel in a field effect transistor structure results in unexpected "normally on" transistorlike behavior. We report here the role of ion migration in transistors based on metallic polymer [poly(3,4-ethylenedioxythiophene) doped with poly(styrene sulfonic acid) (PEDOT:PSS)]. The analysis of the ion flow into the active channel due to the gate voltage suggests that for the transistors studied compensating $\sim 2$ counterions per 100 dopant molecules suppresses PEDOT conductance up to three orders of magnitude. We determine from the temperature dependence of the conductivity of the active channel that the change in conductance of the active channel is throughout the channel in contrast to confinement of gate field induced charge carriers to the channel/dielectric interface in conventional transistors. The decrease of channel conductance reflects an increase of activation energy of carriers. Therefore, we propose that removal of intermediate hopping sites in the disoriented PEDOT:PSS by a small fraction of ion charge compensation causes carriers to anisotropically hop over longer distance leading to a conductor-nonconductor transition.
\end{abstract}

DOI: 10.1103/PhysRevB.74.235219

PACS number(s): 85.30.Tv, 82.35.Cd, 73.61.Ph, 66.30.Dn

\section{INTRODUCTION}

Organic-based materials are of great interest for application in the field effect transistors in electronic circuitry due to the inherent potential of molecule design flexibility, largearea coverage, inexpensive cost, and simplicity of processing. ${ }^{1-5}$ Highly crystalline small molecule systems such as pentacene and $\alpha$-sexithiophene structurally form parallel stacks of molecules with strong overlap of $\pi$ and $\pi^{*}$ orbitals on adjacent molecules forming networks beneficial for charge transport. ${ }^{6,7}$ Field effect mobilities $\left(\mu_{F E T}\right)$ as high as $1.5 \mathrm{~cm}^{2} /(\mathrm{V} \mathrm{s})$ have been reported for the organic semiconductor, pentacene. ${ }^{8,9}$ Those reported values are comparable to that of amorphous Si-based FETs. ${ }^{10}$ Undoped conjugated polymers (CPs) are partially ordered ${ }^{11}$ semiconductors with conductivities in the range from $10^{-10} \mathrm{~S} / \mathrm{cm}-10^{-5} \mathrm{~S} / \mathrm{cm} .{ }^{12}$ The highest mobility reported for an undoped $\mathrm{CP}$ is $\mu_{F E T}=0.28 \mathrm{~cm}^{2} /(\mathrm{V} \mathrm{s})$ for regioregular poly(3-hexylthiophene). ${ }^{13}$ Heavily doped CPs are conductors with the finite densities of states at the Fermi level. ${ }^{12,14-16}$ The conductivities for doped CPs range from $10^{-2} \mathrm{~S} / \mathrm{cm}-10^{5} \mathrm{~S} / \mathrm{cm} .{ }^{12}$ However, the charge transport of this class of organic conductor behaves like neither conventional metals nor semiconductors due to their morphological structure. ${ }^{14,15,17}$

An electrical field effect in devices using the conjugated polymer [poly(3,4-ethylenedioxythiophene) fully doped with poly(styrene sulfonic acid) (PEDOT:PSS)] as the active channel was recently reported. ${ }^{18-21}$ The chemical structure for PEDOT:PSS is shown in Fig. 1. It was later shown that the electrical field effect was common among other doped conducting polymers. ${ }^{19,22}$ For these devices, a potential was applied to a gate electrode spatially separated by an insulating layer from an active channel comprised of a doped conducting polymer. An apparent conductance modulation of the conducting polymer film was observed. ${ }^{18-22}$ The suppression of the conductance of highly doped polymers is unexpected because of the known screening effect common in conductors. ${ }^{19}$ The penetration of external electric field inside the organic conductor is contradictory to the prediction of the Debye screening length for metals. For a metal with free carrier concentration $(n) \sim 10^{21} \mathrm{~cm}^{-3}$, relative permittivity $\sim 100$, and Fermi energy $\sim 1 \mathrm{eV}$, the electric field is screened within $2 \mathrm{~nm}$ of the surface, ${ }^{23}$ which is negligible as compared to the typical thickness $(50-100 \mathrm{~nm})$ of the active channel. Okuzaki et al. ${ }^{21}$ reported $\mu_{F E T} \approx 170 \mathrm{~cm}^{2} /(\mathrm{V} \mathrm{s})$ for hole carriers on the PEDOT backbone by using the conventional FET model ${ }^{24}$ to analyze their data. This value is in contradiction with mobility estimated from the conductivity $(\sigma)$. Using $\sigma=n e \mu_{\sigma}$ yields $\mu_{\sigma} \sim 0.2 \mathrm{~cm}^{2} /(\mathrm{V} \mathrm{s})$ for PEDOT: PSS for $\sigma \sim 30 \mathrm{~S} / \mathrm{cm}$ and $n \sim 10^{21} \mathrm{~cm}^{-3}$. Hence, $\mu_{F E T}$ $>10^{2} \mu_{\sigma}$ and is not a physical result. In addition, this electrical field effect was suppressed by lowering $10{ }^{\circ} \mathrm{C}$ below room temperature. ${ }^{19}$ Further, the field effect response of the active channel is relatively slow. Therefore, Epstein et al. ${ }^{19}$ proposed that ion motion was involved in the conductance modulation of conducting polymer based FETs.

In this paper, we report the role of ions driven by electric field inside a metallic PEDOT:PSS polymer film using a typical device structure illustrated schematically in Fig. 2(a). Evidence for the influence of ion motion in the device operation is in the hysteresis of the drain current $\left(I_{D}\right)$-drain

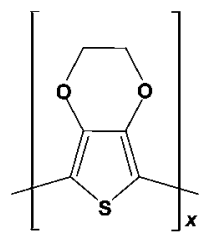

PEDOT

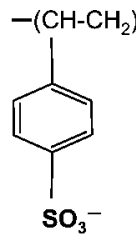

PSS-

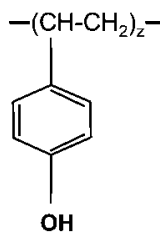

PVP
FIG. 1. The chemical structures for poly $(3,4-$ ethylenedioxythiophene) doped with poly(styrene sulfonic acid) (PEDOT:PSS) and polyvinylphenol (PVP). 


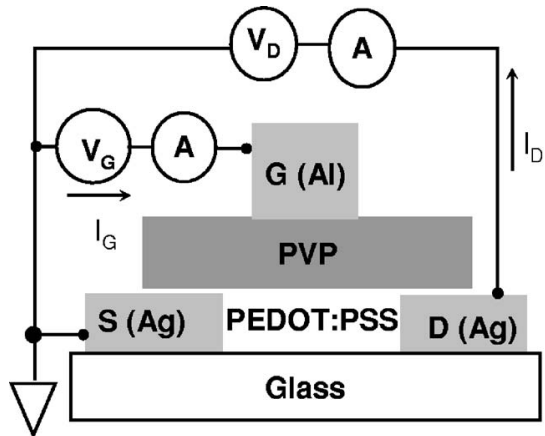

(a)

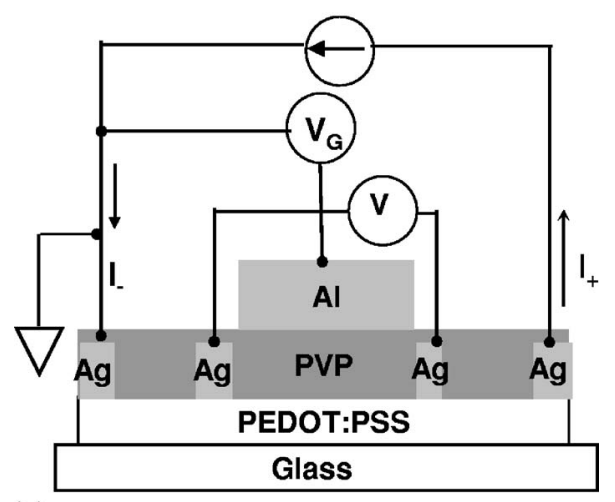

(b)

FIG. 2. Schematic representation for both (a) two-probe and (b) four-probe circuits used in studying the device response to the application of electric field.

voltage $\left(V_{D}\right)$ characteristics and also in transient $I_{D}$ measurements. The ion current injected into the active channel was recorded in the gate current $\left(I_{G}\right)$ through the gate contact $(G)$. The correlation between the injected ion charges and the channel conductance suggests a percolating mechanism for conduction suppression. Devices studied here have a range of behaviors. Generally, 0.01-0.1 ionic charges inserted in every three ring PEDOT repeat produces a significant change in the conductivity of the metallic polymer. We determined that typically approximately $2 \%$ of the holes on the polymer backbone compensated by ionic charges inserted from the insulating layer was sufficient to alter the PEDOT conductance up to three orders of magnitude. This small fraction of charge compensation demonstrates that the suppression of PEDOT:PSS conductance does not result from a pure electrochemical doping-dedoping process. In addition, an increase of activation energy $T_{0}$ with a decrease of PEDOT:PSS conductivity implies that the modulation of active channel conductance is throughout the whole channel and is in accord with the presence of the percolation phenomenon. Furthermore, the increase of $T_{0}$ indicates a reduction of carrier hopping sites on a charge transport path. We proposed that the inserted ions effectively interrupt the low dimensional carrier hopping network on the PEDOT backbone causing a conductor-nonconductor transition. This is associated with the charge transport nature in the inhomogeneous morphological system by interrupting the localized states available for hopping between ordered regions.

\section{EXPERIMENTAL}

Two $50 \mathrm{~nm}$ thick silver (99.9\%) pads were thermally deposited on a glass substrate (precleaned micro slides from Gold Seal ${ }^{\circledR}$ Products) as source $(S)$ and drain $(D)$ contacts to define the channel dimensions followed by spin coating the modified PEDOT:PSS solution across the $S / D$ contacts as the active channel. We mention here that the $I_{D}-V_{D}$ relationship for the $S /$ modified PEDOT:PSS/D junction shows no difference when using $\mathrm{Au}$ as $S / D$ contacts. Glass slides were cleaned with acetone in an ultrasonicator before being used and the spin coating process was carried out by using a photoresist spinner Model 1-PM101DT-R790 from Headway Research Inc. in the laboratory environment inside a fume hood. The original PEDOT:PSS solution $(0.5$ wt. \% PEDOT and 0.8 wt. \% PSS dispersion in water, catalogue number 483095-250G) was purchased from Aldrich Chemicals. The molar ratio for PEDOT to PSS is approximately 1 to 1.25 . In such case, PEDOT is expected to be "fully doped" and have approximately one hole charge for every three PEDOT rings. ${ }^{25}$ The conductivity for the original PEDOT:PSS films was approximately $0.8 \mathrm{~S} / \mathrm{cm}$ by standard four probe method. The modified PEDOT:PSS solution ${ }^{26-28}$ was a mixture of 80 wt. \% PEDOT:PSS, 20 wt. \% ethyleneglycol (99.8\%, Aldrich) and $<0.05$ wt. $\%$ dodecylbenzenesulfonic acid (DBSA) (Acros Organics). The conductivity of the modified PEDOT:PSS films was approximately $30 \mathrm{~S} / \mathrm{cm}$ by standard four probe method as expected for fully doped highly conducting PEDOT:PSS. ${ }^{27}$

After drying the PEDOT:PSS layer in the air for two days, 0.5 wt. \% polyvinylphenol (PVP) $(99.5 \%$, Aldrich) in isopropanel (Aldrich) was spin coated on top of the PEDOT:PSS layer as the dielectric layer. Finally, the aluminum gate electrode $(G)$ was thermally deposited on top of the PVP layer without overlapping with $S / D$ contacts to complete the device fabrication. The thickness for PEDOT:PSS, PVP, and Al ranged from $50 \mathrm{~nm}$ to $85 \mathrm{~nm}, 30 \mathrm{~nm}$ to $50 \mathrm{~nm}$, and $30 \mathrm{~nm}$, respectively. The typical dimensions for the active channel length $(L)$ and width $(W)$ were $500 \mu \mathrm{m}$ and $900 \mu \mathrm{m}$, respectively. Chemical structures for both PEDOT:PSS and PVP are shown in Fig. 1.

The finished device structures were characterized by twoprobe and four-probe methods in the laboratory environment by using colloidal silver paste (Electron Microscopy Sciences, Washington, PA) to attach the Ag and Al electrodes to the circuitry inside a metal-shielded box. The detail of the circuitry is shown in Fig. 2. Both $I_{D^{-}} V_{D}$ and transient $I_{D}$ current studies of the device response were carried out by using two-probe method at room temperature in the laboratory environment. Two Keithley Model 487 meters supplied voltages and recorded current in both $S / D$ and $G / S$ loops. The four-terminal $\rho(T)$ measurements were performed by using a Keithley Model 2400 as the current supply and the voltage meter while a Keithley Model 487 functioned as the gate voltage supply. We kept devices inside a shielding copper box in a Delta Model 9059 environmental chamber with $\mathrm{N}_{2}$ continuous purging environment. An iron-constantan thermal couple was inserted to measure the temperature on top of the device. We applied different magnitudes of current 
to the sample and determined the resistance values from the linear $I(V)$ region.

\section{RESULTS}

\section{A. $I_{D}-V_{D}$ measurements}

The circuitry arrangement shown in Fig. 2(a) was used to characterize the fabricated device structures. With $V_{G}$ fixed at constant values, $V_{D}$ was swept from +5 to $-5 \mathrm{~V}$ and back to $+5 \mathrm{~V}$ at typically $25 \mathrm{mV} / \mathrm{s}$. The measurements were repeated for $V_{G}$ varied discretely by $\Delta V_{G}=0.5 \mathrm{~V}$ from -1 to $+5 \mathrm{~V}$. Output characteristics $\left(I_{D^{-}} V_{D}\right)$ for selected $V_{G}$ 's are shown in Fig. 3(a) for a device with thickness $75 \mathrm{~nm}$ and $50 \mathrm{~nm}$ for PEDOT:PSS and PVP layers, respectively. Hysteresis effects were present for both $V_{D}$ polarities. Applying $V_{G}$ 's to the devices results in dramatically different $I_{D}-V_{D}$ for positive and negative $V_{D}$ polarities. There was no apparent difference of $I_{D}\left(V_{D}\right)$ for $V_{G}<0$ and $V_{G}=0 \mathrm{~V}$. However, applying positive $V_{G}$ substantially altered $I_{D}\left(V_{D}\right)$ in both $V_{D}$ polarities. The conductance of the active channel decreased as $V_{G}>0 \mathrm{~V}$. In particular, there is a more pronounced conductance suppression effect for the $V_{D}<0 \mathrm{~V}$ quadrant. The $I_{D^{-}} V_{D}$ curves in $V_{D}<0 \mathrm{~V}$ quadrant showed some similarities to that of a conventional field effect transistor. Therefore, we carried out a more detail study of $I_{D}\left(V_{D}\right)$ for $V_{D}<0 \mathrm{~V}$ on a device with thickness $83 \mathrm{~nm}$ and $46 \mathrm{~nm}$ for PEDOT:PSS and PVP layers, respectively.

As shown in Fig. 3(b), $V_{D}$ was swept from 0 to $-5 \mathrm{~V}$ and back to $0 \mathrm{~V}$. The measurements were repeated for $V_{G}$ varied discretely by $\Delta V_{G}=0.5 \mathrm{~V}$ from 0 to $-1 \mathrm{~V}$ and back to $+6 \mathrm{~V}$. The drain sweeping rate was $25 \mathrm{mV} / \mathrm{s}$ for $V_{G} \leqslant 0 \mathrm{~V}$ and $10 \mathrm{mV} / \mathrm{s}$ for $V_{G}>0 \mathrm{~V}$. The linear relationship between $I_{D}$ and $V_{D}$ in the gate electrode floating condition indicates that both contacts between PEDOT:PSS and Ag electrodes are ohmic. This metal/organic conductor/metal junction behaves as a simple resistor with no hysteresis observed for $\Delta V_{D}$ up to $1 \mathrm{~V} / \mathrm{s}$ (the maximum rate we used). Applying $V_{G}$ 's substantially changed the $I_{D}-V_{D}$ characteristics. Both linear and saturation regions occur at $V_{G}>0 \mathrm{~V}$. Increasing $V_{G}$ 's decrease the channel conductance and eventually strongly suppresses the charge conduction. The data shown in Fig. 3(b) shows a maximum factor of 10 decrease in the channel conductance while other devices using a different configuration $^{29}$ display up to $10^{5}$ fold suppression of channel conductance at $V_{G}=+20 \mathrm{~V} .{ }^{30}$ Utilizing the conventional FET model, ${ }^{21,24}$ the analysis shown in the inset of Fig. 3(b) yields $\mu_{F E T} \approx 52 \mathrm{~cm}^{2} /(\mathrm{V} \mathrm{s})$. As discussed in the Introduction, this value is not a physical result. We note that the hysteresis occurs during sweeping $V_{D}$ 's even at sweeping rate as low as $10 \mathrm{mV} / \mathrm{s}$. This suggests a role of ion motion in the charging-discharging phenomenon.

\section{B. Transient $I_{D}$ study}

The transient $I_{D}$ study provides information of the role of ions in the PEDOT:PSS conduction modulation. Initially, $V_{D}$ and $V_{G}$ were kept at $+0.1 \mathrm{~V}$ and $0 \mathrm{~V}$, respectively. We then applied a positive $V_{G}$ for $30 \mathrm{sec}$ before resetting $V_{G}$ to $0 \mathrm{~V}$. The applied $30 \mathrm{sec} V_{G}$ pulses ranged from 0 to $+4 \mathrm{~V}$ in
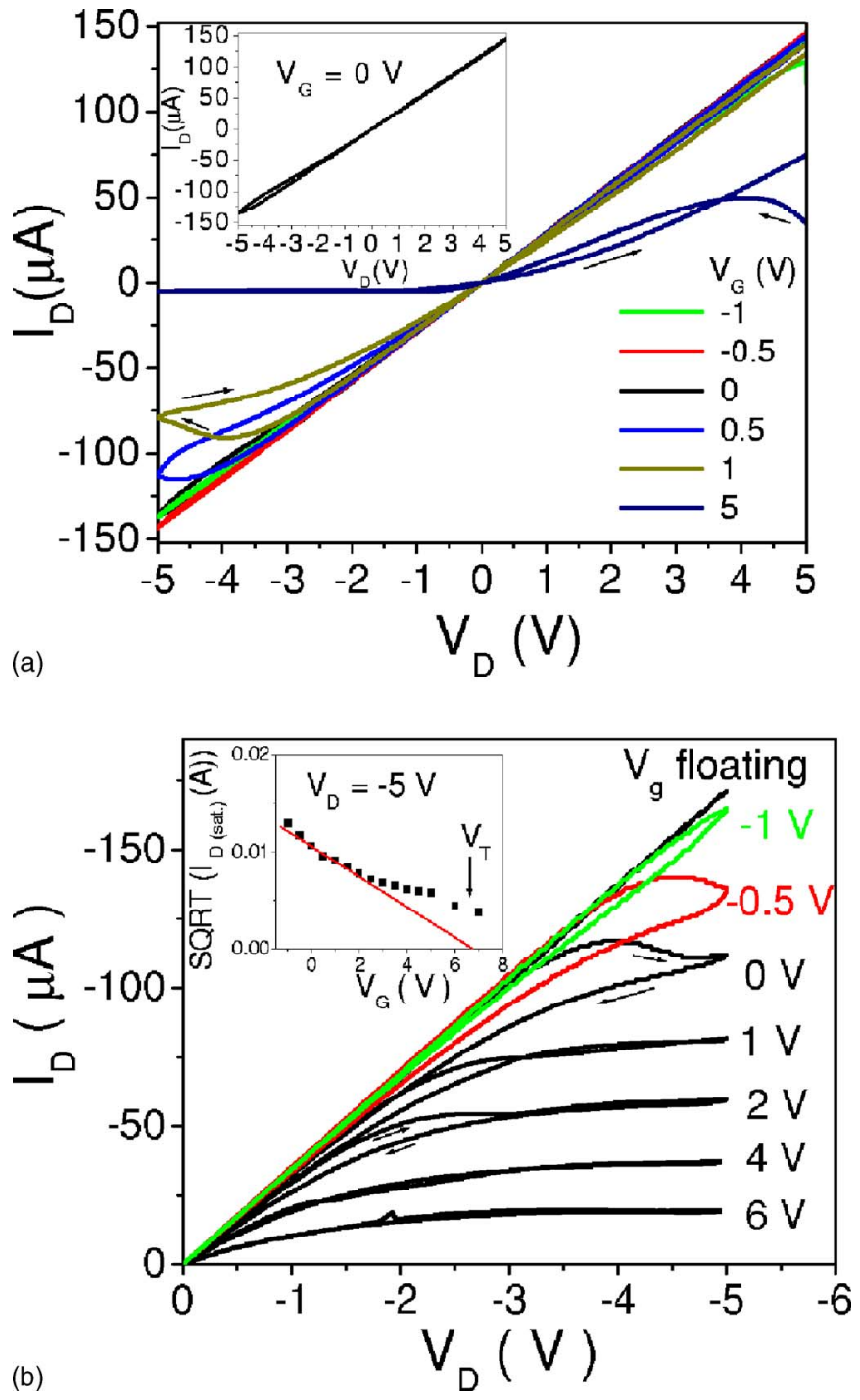

FIG. 3. (Color online). Hysteresis $I_{D^{-}} V_{D}$ characteristics for different applied gate voltages. (a) Results for selected $V_{G}$ 's are shown in both $V_{D}>0 \mathrm{~V}$ and $V_{D}<0 \mathrm{~V}$. The order for the application of gate voltages is from $-1 \mathrm{~V}$ to $+5 \mathrm{~V}$. The inset is the result for $\mathrm{V}_{\mathrm{G}}=0 \mathrm{~V}$. (b) Results for $V_{D}<0$ for a different device with the application of $V_{G}$ 's from $0 \mathrm{~V}$ to $-1 \mathrm{~V}$ and then back to $+6 \mathrm{~V}$. The inset shows the square root of $I_{D}$ at the saturation region $\left(I_{D}\right.$ (sat.) $)$ vs $V_{G}$. According to the traditional FET model, ${ }^{24}$ both the threshold voltage $\left(V_{T}\right)$ and $\mu_{\mathrm{FET}}$ can be obtained and calculated from the red fitting curve.

steps of $0.5 \mathrm{~V}$. The current through the PEDOT:PSS active channel was measured and is displayed in Fig. 4(a). Apparently, the $I_{D}$ has not reached a static state within $30 \mathrm{sec}$ and its recovery time after resetting $V_{G}$ to $0 \mathrm{~V}$ takes even longer. In each $V_{G}>0 \mathrm{~V}$ period, the initial channel conductance varies linearly with time. We describe the initial time dependence of the drain current as

$$
I_{D}(t)=I_{D}(0)(1-k t)
$$

where $k$ is the $V_{G}$ dependent response rate of PEDOT:PSS and $I_{D}(0)$ is the drain current level just before $V_{G}$ is applied. 

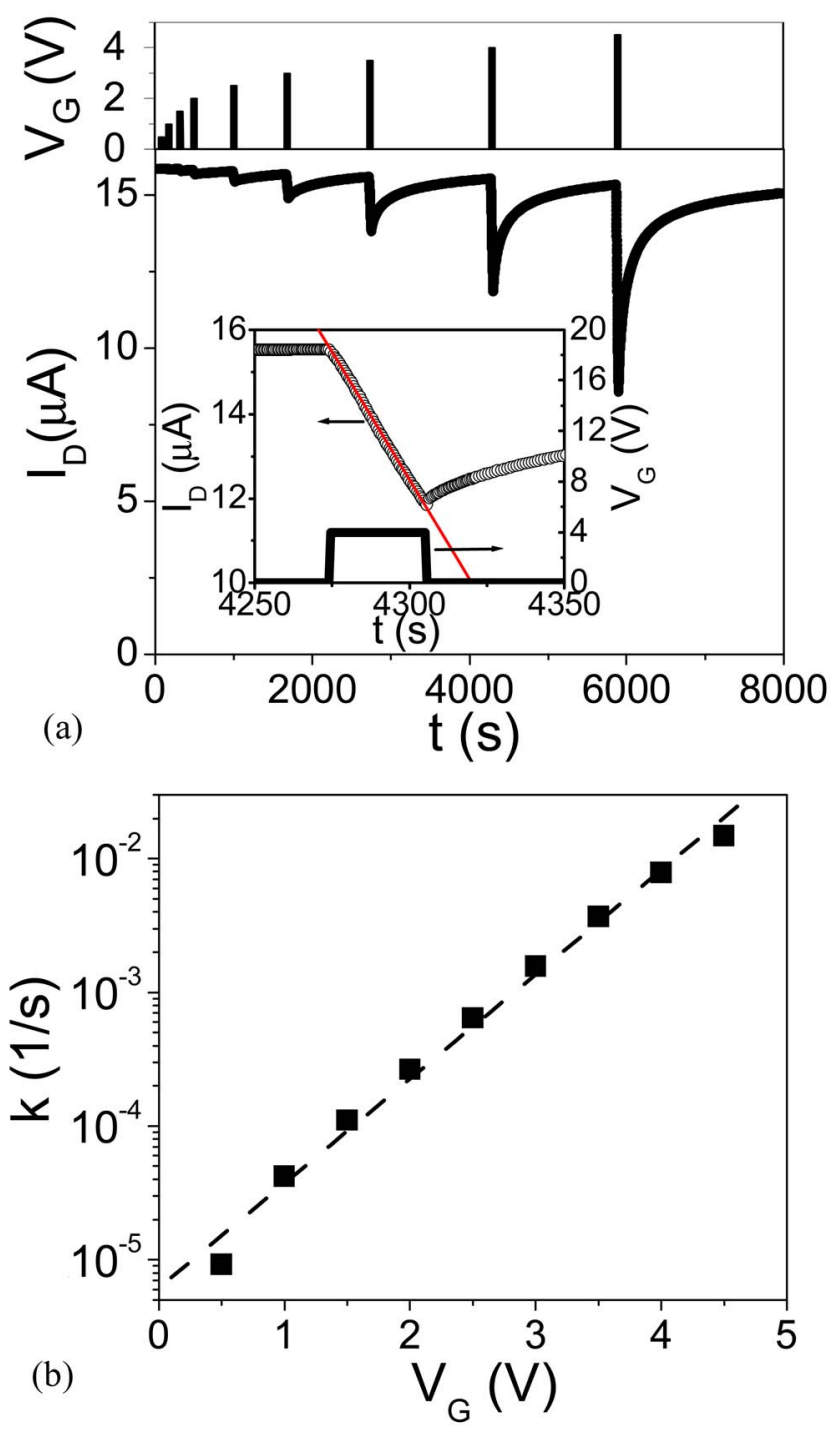

FIG. 4. (Color online). The conductance response of the active channel by the application of 30-second gate voltage pulses. (a) $I_{D}$ modulation by the $30 \mathrm{sec} V_{G}$ pulses. The top panel shows the modulation profile of $V_{G}$ 's ranging from $0 \mathrm{~V}$ to $+4.5 \mathrm{~V}$ and the bottom one is the corresponding $I_{D}$. The expanded graph of eighth cycle $\left(V_{G}=+3.5 \mathrm{~V}\right)$ is shown in the inset. (b) The response rate $(k)$ of $I_{D}$ vs $V_{G}$.

By fitting Eq. (1) to various $V_{G}>0$ periods, we obtain $k\left(V_{G}\right)$. These values are plotted in Fig. 4(b), showing exponential dependence of $k$ on $V_{G}$

$$
k=k_{0} \exp \left(V_{G} / V_{0}\right) .
$$

The dashed fitting line in Fig. 4(b) yields $k_{0}=6 \times 10^{-6}(1 / \mathrm{s})$ and $V_{0}=0.556 \mathrm{~V}$ for the experimental results obtained in Fig. 4(a). For conventional metal-oxide FETs (MOSFETs), the response rate for $I_{D}$ is determined by carrier mobility which does not show the exponential dependency on gate electric field. ${ }^{31}$ As proposed in Ref. 19 , the obtained $k\left(V_{G}\right)$ is associated with the voltage dependence of ion hopping frequency inside PEDOT:PSS. For ion hopping inside a polymer system, possible origins for changing conductance are (1)

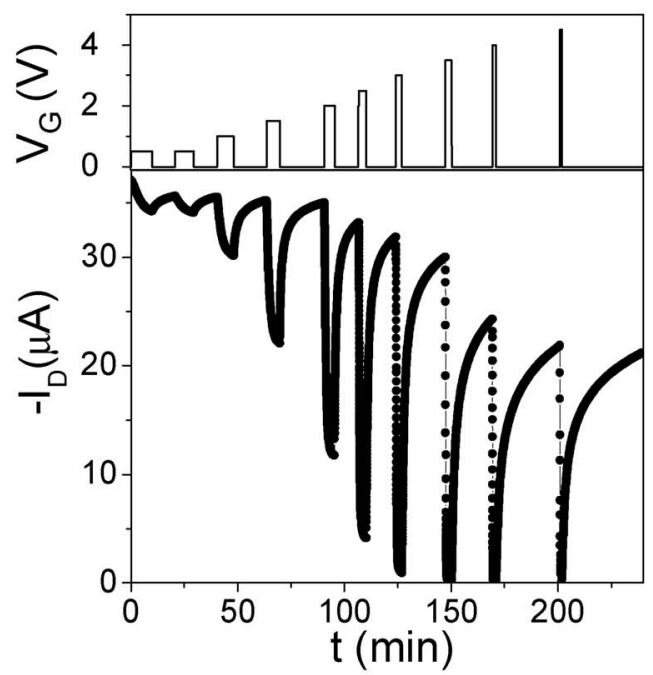

(a)

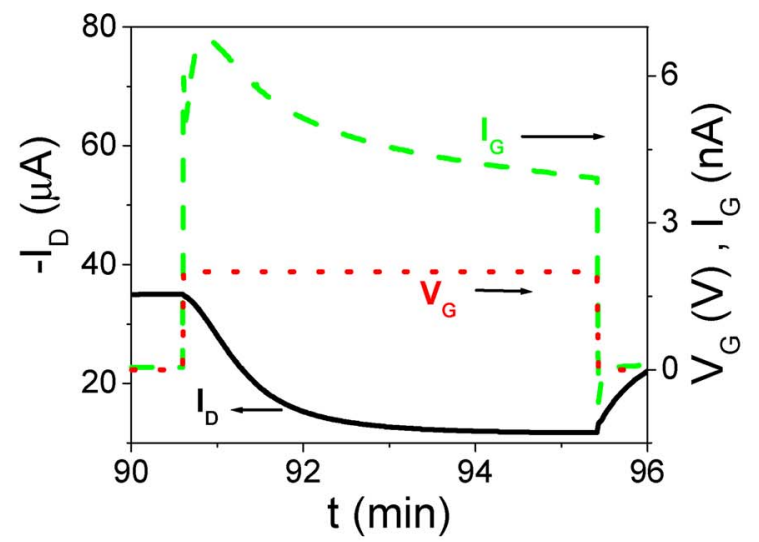

(b)

FIG. 5. (Color online). (a) The modulation of $I_{D}$ by application of $V_{G}$ 's for long time periods. The modulation profile of $V_{G}$ 's is shown on the top panel. A zoom in cycle for $V_{G}=+2 \mathrm{~V}$ including $I_{G}$ is shown in (b). Data for $V_{G}, I_{D}$ and $I_{G}$ are collected every $0.5 \mathrm{sec}$.

changing free carrier concentration through electrochemical doping-dedoping ${ }^{32}$ and (2) changing effective carrier mobility. The detailed mechanism will be discussed in the Discussion section.

To further probe ion motion in a conducting polymer matrix, the experiment on Fig. 4(a) was repeated but with a longer period of positive $V_{G}$ pulse imposed. The initial and final states of the polymer upon applying $V_{G}$ were studied by the following experiment. $V_{D}$ was kept at $-1 \mathrm{~V}$ and $V_{G}$ pulses were applied from $0 \mathrm{~V}$ to $+4.5 \mathrm{~V}$ in $0.5 \mathrm{~V}$ steps. A positive $V_{G}$ was applied until the $I_{D}$ variation rate was less than $20 \mathrm{nA} / \mathrm{min}$ followed by resetting $V_{G}$ to $0 \mathrm{~V}$ until approximately recovery of $I_{D}$ to $90 \%$ of its initial value. Both the modulated $I_{D}$ and $I_{G}$ were monitored continuously as shown in Fig. 5. We measured the current through the gate contact of less than few tens nA for $V_{G}>0 \mathrm{~V}$ and less than $1 \mathrm{nA}$ at $V_{G}=0 \mathrm{~V}$ for these devices. As an example, Fig. 5(b) shows the reduction of $I_{D}$ at $V_{G}=+2.5 \mathrm{~V}$ as well as the variation of $I_{G}$ recorded simultaneously with $I_{D}$. The variation of 

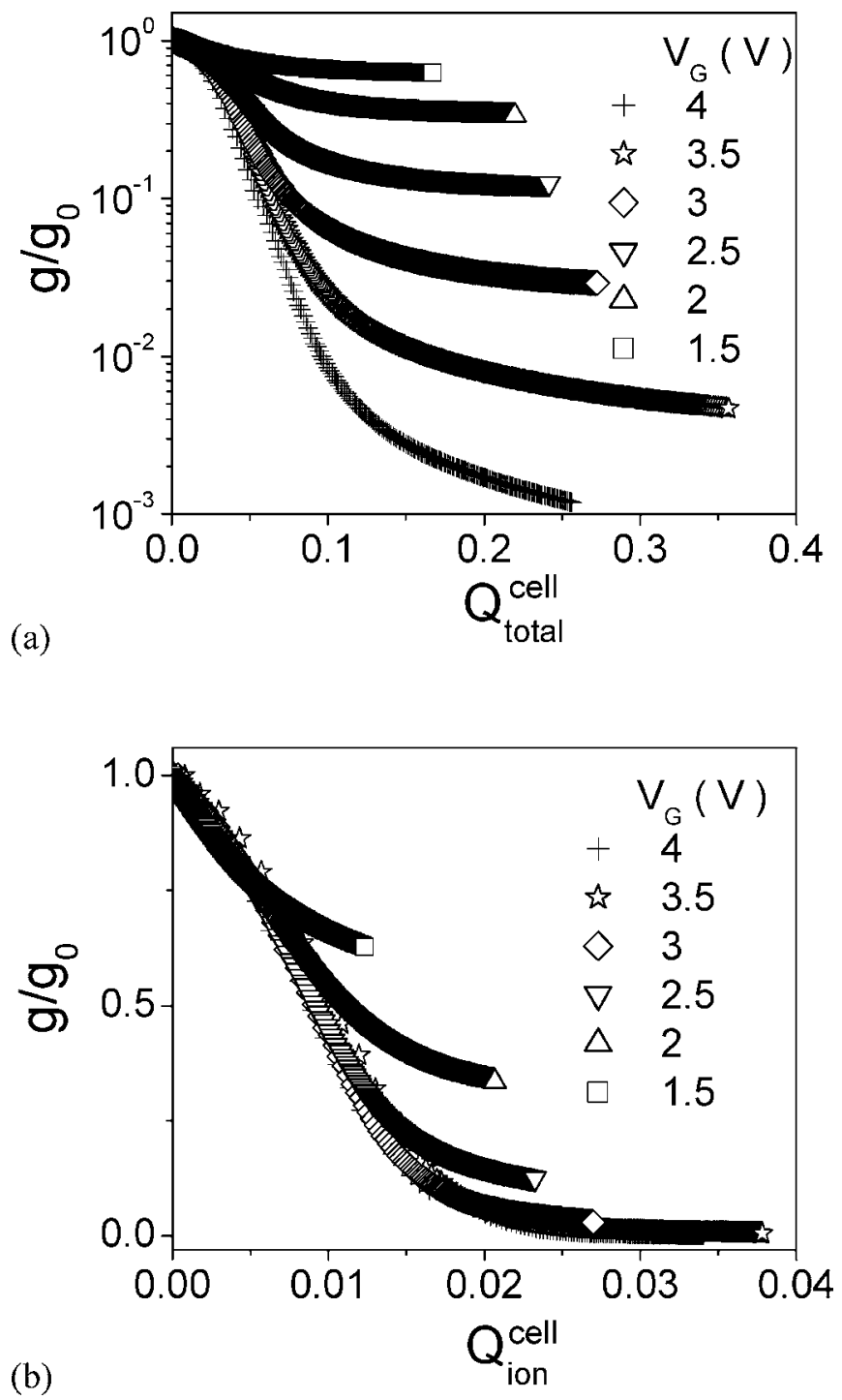

FIG. 6. (a) The evolution of channel conductance $\left(g / g_{0}\right)$ as a function of total amount of gate charges injected into the active channel per PEDOT cell $\left(Q_{\text {total }}^{\text {cell }}\right)$ for different $V_{G}$ 's. (b) Reploting of (a) after subtracting the electronic leakage current.

channel conductance approaches equilibrium after applying $V_{G}$ for a longer period of time. This implies that this electron-ion coupled system goes to different final equilibrium configurations at different applied $V_{G}$ 's. The recorded $I_{G}$ shows an initial rapid increase to a rapid decrease followed by a slow increase to a maximum before a substantially decrease to an asymptotic value. This feature of $I_{G}$ is typical in $V_{G}>0$ cycles only and the details vary with $V_{G}$ 's. By resetting $V_{G}=0,\left|I_{G}\right|$ became negative and decayed rapidly before crossing zero to an asymptotic value of less than $80 \mathrm{pA}$.

Since $I_{G}$ is always present during the application of $V_{G}$ and substantially larger than that for Si-based MOFETs, ${ }^{33}$ we study the effect of injected gate charges on the conductance of the active channel $\mathrm{g}$. At time $t$, the total amount of charge injected through the gate into the active channel per PEDOT cell ${ }^{34}$ is $Q_{\text {total }}^{\text {cell }}(t)$, where $Q_{\text {total }}^{\text {cell }}(t)=\int_{0}^{t} I_{G}\left(t^{\prime}\right) d t^{\prime} /(q$ $\times$ Number of PEDOT cells),$q=1.6 \times 10^{-19} \mathrm{C}$. This amount

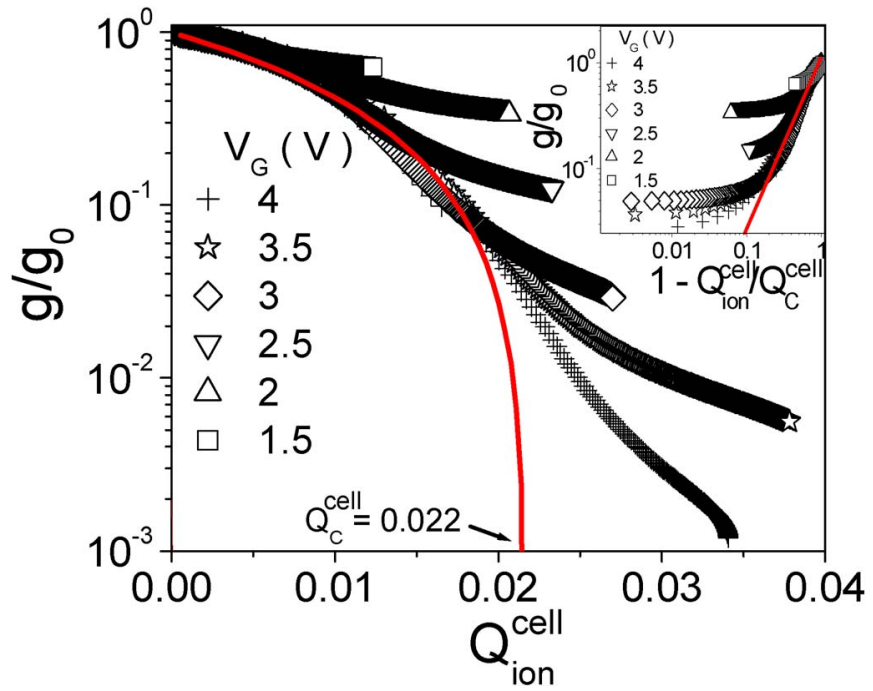

FIG. 7. (Color online). The scaling relation fitting of $g / g_{0}$ vs $Q_{i o n}^{c e l l}$. The inset is the logarithm plot. Red curves are fitting results.

of charge injected through the gate electrode causes a factor of $g(t) / g_{0}$ decrease in the channel conductance, where $g_{0}[=g(0)]$ is the channel conductance at $Q_{\text {total }}^{\text {cell }}(t)=0$. Therefore, we plot $g\left(Q_{\text {total }}^{\text {cell }}\right) / g_{0}$ and the results for the application of different $V_{G}$ 's are displayed in Fig. 6(a).

For a specific $V_{G}$ in Fig. 6(a), the initial injection of gate charge correlates with the suppression of the channel conductance. Injection of additional gate charge has progressively smaller effect on the channel conductance. This suggests that $I_{G}$ has two components, i.e., one remains constant and the other decays rapidly with time [see the upper panel of Fig. 5(b)]. We observed that the peak of $I_{G}$ and the time for $I_{G}$ to reach an asymptote decreased strongly with decreasing temperature. The detail experimental results for $I_{G}$ will be in a separate publication. In addition, our earlier study ${ }^{19}$ showed that the $I_{D}$ modulation was dramatically reduced by the reduction of temperature $10{ }^{\circ} \mathrm{C}$ from room temperature. Therefore, we assign the slow increase and decay feature of $I_{G}$ to the ionic component of $I_{G}$ associated with the ion diffusion motion. The constant (asymptotic) component is the electronic "leakage current." The electronic leakage current is less than $10 \mathrm{nA}\left(V_{G}=4 \mathrm{~V}\right)$ for the data shown in Fig. 5(a). The role of ionic current is also supported in the asymmetrical slow response of charging and discharging phenomenon in $I_{D}$ modulation.

We subtract the background component of constant electronic current and replot $g\left(Q_{i o n}^{\text {cell }}\right) / g_{0}$, where $Q_{i o n}^{\text {cell }}$ is the amount of inserted ion charges injected into the active channel per PEDOT cell. We obtained in Fig. 6(b) that all curves corresponding to different $V_{G}$ 's nearly merge into one curve and this suggests a percolation phenomenon. According to the scaling relationship based on a percolation phenomenon, the conductance of the system is described as ${ }^{35}$

$$
g \sim\left(Q_{C}^{\text {cell }}-Q_{\text {ion }}^{\text {cell }}\right)^{t},
$$

where $Q_{C}^{\text {cell }}$ is the percolation threshold, and $t$ is the dimensionless index. The best fitting result for the obtained merged 
curve by using Eq. (3) is shown in Fig. 7. We obtain $Q_{C}^{\text {cell }}$ $\approx 0.022 \pm 0.001$ and $t \sim 1.41 \pm 0.11$. We confirm these obtained values by plotting in logarithm scale as shown in the inset in Fig. 7 and we obtain a good merge of different curves though partial deviations occur. The deviations from the fit are presumably due to the detail of polymer chain cutting process at different $V_{G}$ 's. The asymptote value for each deviation decreases monotonically with the increase of $V_{G}$.

We determine from $Q_{C}^{\text {cell }}$ that approximately 2 counterions per 100 dopants being compensated by inserted ions is sufficient to cut the conduction paths nearly completely and suppress the conductance more than three orders of magnitude. The continuous decrease of $g$ for $Q_{i o n}^{\text {cell }}>Q_{C}^{\text {cell }}$ may reflect cutting of residual conduction paths as ions reach the previous uncut chains after the polymer lattice starts to respond to the ingress of the inserted ions. The residual conductance (asymptote value) is contributed from the partially cut conducting paths and this can be seen in the temperature dependent measurements.

\section{Temperature dependence of electric field effect on active channel resistivity}

A four-probe configuration in the active channel area was designed to study intrinsic properties of the active channel under the application of gate electric field. For devices studied in this section, we used the same processing conditions and solution formula as mentioned in the experimental section except the device configuration [Fig. 2(b)]. Instead of starting with source/drain electrodes deposition, we spin coated PEDOT:PSS layer (50-85 nm thick) and allowed it air dry for two days followed by thermal deposition of four $200 \mu \mathrm{m}$ wide $30 \mathrm{~nm}$ thick silver probes for performing the resistivity measurement. After that, the PVP layer (30-80 $\mathrm{nm}$ thick) was spin coated two days before thermal deposition of $25 \mathrm{~nm}$ thick $\mathrm{Al}$ as the gate electrode. The gate electrode overlapped at least $90 \%$ of the PEDOT:PSS area between the inner two voltage probes. Explicitly, it does not overlap either electrode. The dimensions for the active channel of the finished devices are $L=1 \mathrm{~mm}$ and $W=0.9 \mathrm{~mm}$. The circuit arrangement in Fig. 2(b) was used to perform the active channel resistivity measurements. The contact resistance was measured to be less than $3 \%$ of the channel resistance.

The measuring sequences used were as follows: We first measured the room temperature channel resistivity $\rho_{0}$ at $V_{G}$ $=0 \mathrm{~V}$. Remaining at room temperature, a positive $V_{G}$ in the range of $2-5.5 \mathrm{~V}$ was applied to the device until $I_{D}$ reached a static state with resistivity value $\rho_{\alpha}$, a factor of $\alpha($ $\left.=\rho_{\alpha} / \rho_{0}\right)$ increase after the application of $V_{G}$. We maintained $V_{G}$ and measured $\rho_{\alpha}(T)$ from $300 \mathrm{~K}$ to $80 \mathrm{~K}$ with $\Delta T$ $=10 \mathrm{~K}$ or $20 \mathrm{~K}$ steps. The cooling rate for each temperature step was less than $0.75 \mathrm{~K} / \mathrm{min}$. Right after setting each temperature, we used the two outer most silver probes as source/ drain electrodes to monitor the channel conductance until it reached an asymptote. Then, we switched back to the four probe circuit to do resistivity measurements before setting the next temperature. After finishing the measurement at
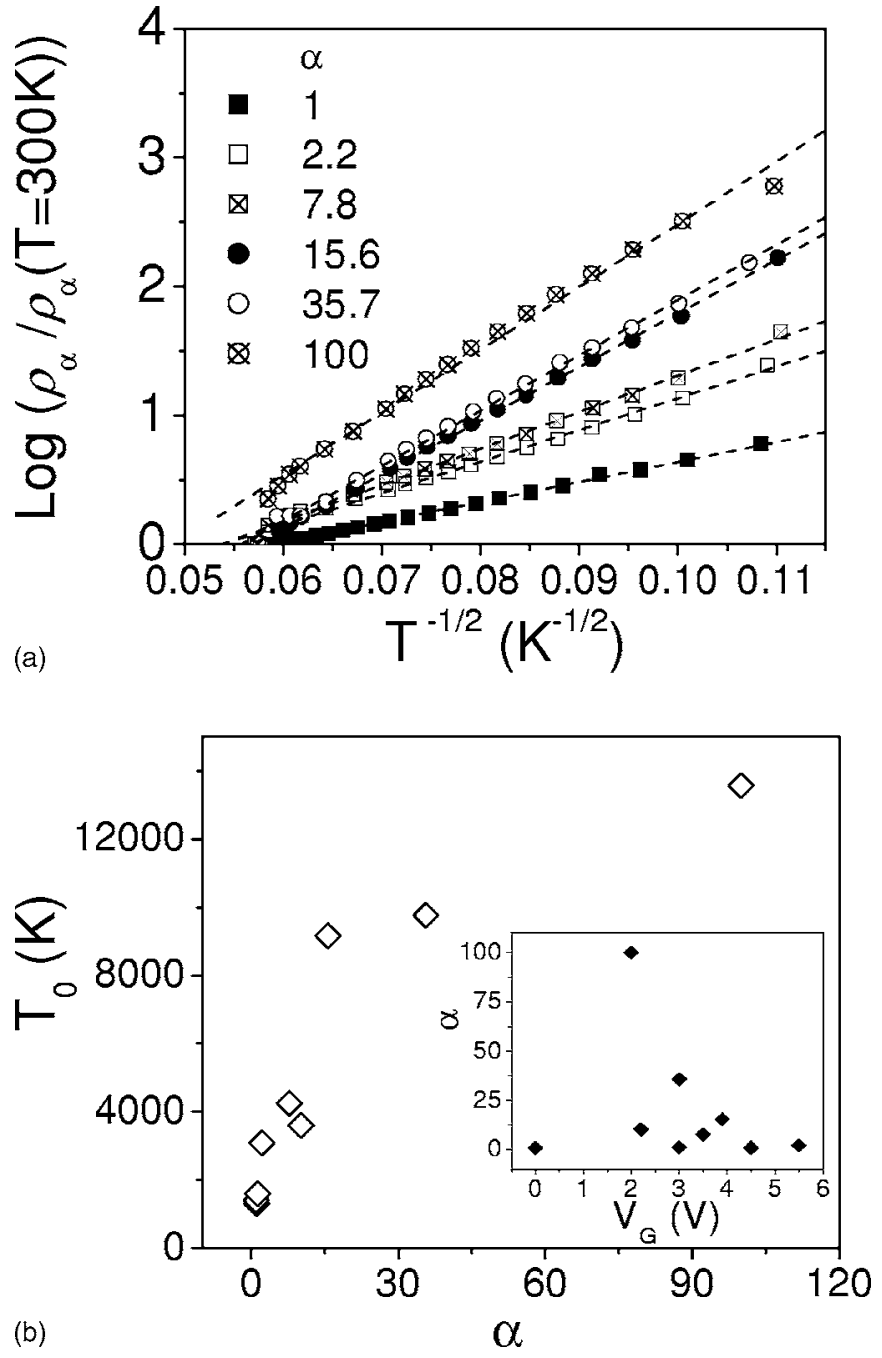

FIG. 8. (a) Quasi-1D VRH Mott's law fitting for temperature dependent channel resistivity measurement at different channel conductive states characterized by $\alpha$. We select 6 data sets for a clear presentation in this chart. (b) $T_{0}$ vs $\alpha$ from $\rho(T)$ measurements. The inset shows $\alpha$ vs. $V_{G}$ for different devices.

$80 \mathrm{~K}$, we immediately warmed up the sample back to $300 \mathrm{~K}$ at a rate of less than $5 \mathrm{~K} / \mathrm{min}$. The channel resistivity was recovered within $3 \%$ of the original state after return back to $300 \mathrm{~K}$. We then reset $V_{G}=0 \mathrm{~V}$ and we found that the active channel resistivity did not completely recover. We continued to apply $V_{G}$ with $0.5-1 \mathrm{~V}$ higher to the same device, the device showed a relatively smaller change in active channel conductance. One of the possible explanations is that the PVP/PEDOT:PSS interface morphology might change after both materials undergoing contraction and expansion. We then used fresh samples to repeat the measurement procedures mentioned above with different values of $\alpha$. We mention here that the strength of the field effect in the active channel for devices using an ionic conductor, polyethylene oxide: lithium perchlorate, as dielectric layer is more reproducible after low temperature operation. ${ }^{36,37}$

We tried to plot $\alpha$ vs $V_{G}$ for different devices used in resistivity measurements, yet no monotonic relationship was found [the inset in Fig. 8(b)]. This might be possibly due to 
a subtle various in PVP thickness and different PVP/ PEDOT:PSS interface morphology causing differences in ion distribution. In order to study the effect of $V_{G}$ on bulk resistivity of the active channel, we examine the behavior of $\rho_{\alpha}(T) / \rho_{\alpha}(T=300 \mathrm{~K})$ and we found that there is a monotonic behavior in comparing the intrinsic property of the active channel.

The $T$ dependence of $\rho_{\alpha}(T) / \rho_{\alpha}(T=300 \mathrm{~K})$ is presented in Fig. 8(a), where $\rho_{\alpha}(T)$ is plotted versus $T^{-1 / 2}$ on a log-linear scale. Mott's $\operatorname{law}^{38} \rho_{\alpha}(T)=\rho_{0} \exp \left[\left(T_{0} / T\right)^{1 / 2}\right]$ for quasi-onedimensional variable range hopping (1D VRH) transport in a disordered system holds ${ }^{39}$ for each $\alpha$ state. The activation energy $T_{0}$ is related to the localization length $(\xi)$ and density of states at the Fermi level $\left[N\left(E_{F}\right)\right]$ through $T_{0}$ $=c /\left[k_{B} N\left(E_{F}\right) \xi\right]$ for a quasi-1D system, where $c$ is the proportionality constant and $k_{B}$ is the Boltzmann constant. The calculated $T_{0}$ from the slope of the $\log \left[\rho_{\alpha}(T) / \rho_{\alpha}(300 \mathrm{~K})\right]$ vs $T^{-1 / 2}$ for each $\alpha$ is presented in Fig. 8(b). A gradually increase of $T_{0}$ with the increase of $\alpha$ was obtained.

\section{DISCUSSION}

The field effect transistor structure using metallic polymers as the active channel results in an unexpected normally "on" transistorlike behavior. The penetration of electric field inside a metallic polymer contradicts the charge screening effect expected for a metal with a partially filled energy band. In addition, there is a significant time lag between the applied gate voltage and the drain current response. Further, the electric field effect in metallic polymers is severely attenuated below room temperature. ${ }^{19}$ It is proposed in Ref. 19 that the origin of this novel phenomenon is insertion and subsequent motion of a small density of ions. Here, we study ion motion induced novel behavior in metallic polymer transistors more critically.

The switching rate for field effect transistors is determined by the $R C$ time constant $(\tau)$ of the gate metal electrode/dielectric/active channel capacitor. For the devices studied here, the calculated capacitance is approximately $570 \mathrm{pF}$ for active channel area $500 \mu \mathrm{m} \times 900 \mu \mathrm{m}$ and $50 \mathrm{~nm}$ thick PVP layer (relative permittivity $\sim 5.8$ measured from a Al/PVP/PEDOT:PSS capacitor). $R \approx \frac{V_{D} / 2}{I_{G}} \approx 1 \mathrm{G} \Omega$ using $I_{G}$ at $V_{G}=0 \mathrm{~V}$ and $V_{D}=-0.05 \mathrm{~V}$ from $I_{D}-V_{D}$ curve. The calculated $R C$ time constant is less than $0.57 \mathrm{~s}$. Hence, there is no expectation of an asymmetric slow current switching dynamic in the real time domain for a conventional electric field effect. The experimental switching time of the order of minutes for the metallic transistors reflects the central role of the motion of ion species with mobility much lower than that expected for positive hole charges on the polymer backbone $\left[0.2 \mathrm{~cm}^{2} /(\mathrm{V} \mathrm{s})\right]$.

The participation of mobile ions is reflected in $I_{D^{-}} V_{D}$ curves (Fig. 3) as well. In Fig. 3(b), the active channel behaved as a simple resistor showing no hysteresis effect up to the lateral electric field strength $2 \times 10^{3} \mathrm{~V} / \mathrm{m}$ before closing the $G$-S circuit. In the presence of finite $V_{D}$ 's, the closed $G-S$ loop produced an electric field perpendicular to the active channel. As mentioned in the Introduction, this electric field is screened within $2 \mathrm{~nm}$ by hole charges inside PEDOT:PSS. ${ }^{23}$ The introduction of a vertical electric field through the PVP layer (on the order of $10^{7} \mathrm{~V} / \mathrm{m}$ ) produces hysteresis in the modulation of lateral conductance of the PEDOT:PSS layer. At the same time, the conductance of the active channel decreases. By decreasing the vertical field strength through the application of $V_{G}<0$, the active channel conductance gradually recovered to the floating gate resistance with smaller $I_{D}-V_{D}$ hysteresis effect compared to that for $V_{G}=0$. This suggests that the movement of mobile ions in the direction perpendicular to the drain current flow initiates the hysteresis effect and conductance suppression.

The detailed movement of mobile ions perpendicular to the active channel is suggested by the results in Fig. 3(a). Assuming only PEDOT:PSS film contains mobile impurities, both anions and cations respond to electric field accordingly. At $V_{G}=0$, the vertical electric field strength inside PVP is the same along the active channel for both $V_{D}$ polarities except for the electric field directions. According to the inset in Fig. $3(\mathrm{a})$, hysteresis effect occurred when the ion motion started to appear at $V_{D}<-2 \mathrm{~V}$, but no similar behavior was recorded for $V_{D}>+2 \mathrm{~V}$. This asymmetric driving of the same amount of opposite charged particles originally present inside PEDOT:PSS by the same electric field strength but opposite direction is not physically possible. A possible explanation for the hysteresis effect is the involvement of mobile ions for $V_{D}<-2 \mathrm{~V}$ from the external sources other than PEODT:PSS. These mobile ions start to be driven into the active channel at average field strength on the order of $10^{7} \mathrm{~V} / \mathrm{m}$ across the PVP layer. This is supported by other sets of $I_{D}\left(V_{D}\right)$ comparisons. From $I_{D}\left(V_{D}>0\right)$ at $V_{G}=-1 \mathrm{~V}$, there was a relative small increase for the active channel conductance compared to $V_{G}=0 \mathrm{~V}$. However, apparent conductance suppression was obtained in $I_{D}\left(V_{D}<0\right)$ at $V_{G}=$ $+1 \mathrm{~V}$. In addition, the latter case demonstrated a more pronounced hysteresis effect than the former one. Thus, for the same vertical strength inside PVP, the electric field with direction pointing to gate electrode shows less active ion motion than electric field directed away from the gate electrode. This result indicates that ions (if there are) inside PEDOT:PSS are relatively bounded. Mobile ions involved in the pronounced channel conductance modulation are cations and mostly driven into the active channel through PVP/ PEDOT:PSS interface. Sandberg et al. ${ }^{40}$ and Bäcklund et $a l .{ }^{41}$ have reported that mobile impurity ions (e.g., $\mathrm{H}^{+}, \mathrm{Na}^{+}$, $\left.\mathrm{Cl}^{-}, \mathrm{OH}^{-} \ldots\right)$ can diffuse/drift inside the insulating layer PVP or through $\mathrm{PVP} /$ regioregular poly(3-hexylthiophene) interface to dope/dedope the semiconducting regioregular poly(3hexylthiophene) channel. As reported in Refs. 40 and 41, ion diffusion/drift motion causes the slow switch rate on the order of seconds, hysteresis and high gate current on the order of nA. These observations are similar to those observed in this study.

The movement of mobile ions is qualitatively recorded in $I_{G}$. For devices studied, $I_{G}$ was measured on the order of nA as an example shown in Fig. 5(b). We observed an initial spike at the moment of applying a positive value of $V_{G}$ and this spike came from the charging of Al/PVP/PEDOT:PSS capacitor, which is related to the $R C$ time constant. Typically $R C$ is less than $0.3 \mathrm{~s}$ which is shorter than the data acquisi- 

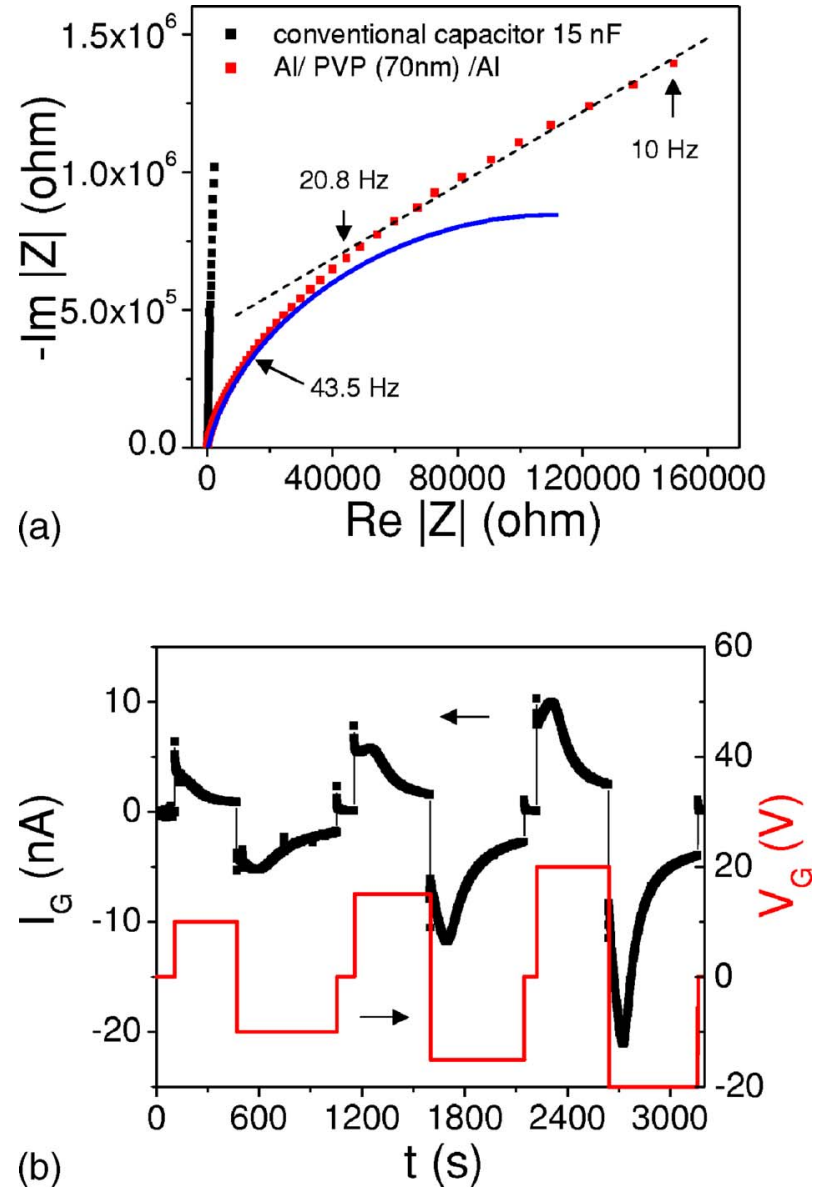

FIG. 9. (Color online). Characterization of an Al/PVP/Al structure. (a) Impedance spectroscopy of an Al/PVP/Al structure with $70 \mathrm{~nm}$ thick PVP layer (red curve) and a conventional capacitor (black curve). The dash line is the linear fitting line and the blue curve is the guideline for a semicircle. (b) dc current response for $400 \mathrm{~nm}$ thick PVP layer. The electric field strength across PVP layer is on the order of $10^{7} \mathrm{~V} / \mathrm{m}$.

tion rate of the instrument limitation. At longer time, the mobile ions started to diffuse across the PVP/PEDOT:PSS interface resulting in a maximum value in $I_{G}$. The time to reach this maximum value depends on the strength of $V_{G}$ which drives ions. Finally, $I_{G}$ decreases and approaches to an asymptotic value. The measured $I_{G}$ in this study shows similarities to that observed by Otero et $a l .{ }^{42}$ through directly monitoring the ionic current in reducing and oxidizing a conducting polymer. As $V_{G}$ reset to $0 \mathrm{~V},\left|I_{G}\right|$ became negative and decay rapidly before crossing zero to an asymptotic value, an electronic leakage current. At the same time, the drain current was gradually recovered. The negative $I_{G}$ indicates the back diffusion of inserted ion charges. A comparison was done using the insulator coated copper wire ${ }^{43}$ as the gate dielectric and gate electrode. In this structure, we did not observe any apparent conductance change of the PEDOT:PSS film with field strength $5 \times 10^{7} \mathrm{~V} / \mathrm{m}$ across the insulator and the $I_{G}$ was simply a spike charging the copper/ insulator/PEDOT:PSS capacitor. This comparison again suggests that ions, if inside the copper coating and PEDOT:PSS, are too bound to be driven by the gate electric field. Thus, it is necessary to inject ion charges into the PEDOT:PSS channel to achieve conductance modulation. Therefore, for studied devices, the current through the channel $\left(I_{D}\right)$ is electronic but the mechanism modulating the current is the ionic movement across the insulator-conducting polymer interface.

To quantify the mobile ion concentration inside PVP layer, we characterized Al/PVP/Al structures by doing impedance spectroscopy in the audio frequency regime and measuring the dc current response under various voltages imposed (see Fig. 9). The impedance spectroscopy for an Al/PVP/Al structure with $70 \mathrm{~nm}$ thick PVP layer was performed by using QuadTech 7600 LRC meter [see Fig. 9(a)]. Unlike the commercially available capacitor (black curve), the results for an Al/PVP/Al structure is comprised of a linear and a portion of a semicircle regions. The low frequency linear region is due to the diffusion of redox pairs (or mobile ions), while the higher frequency semicircle portion originates from the electron transfer at the electrodes. The ion flow is supported by the dc current measurement. Instead of a spike charging current $(R C<0.03 \mathrm{~s})$, the feature for the measured dc current response for an Al/PVP/Al structure (400 nm thick PVP layer) is similar to $I_{G}$ [see Fig. 9(b)]. The concentration for impurities inside PVP is calculated to be on the order of $10^{19} \mathrm{~cm}^{-3}$, two orders of magnitude less than the free carrier concentration in the active channel.

When mobile ions diffuse into the PEDOT active channel layer, a possible outcome is to dedope PEDOT:PSS $\left(\mathrm{PEDOT}^{+} \mathrm{PSS}^{-}+M^{+}+e^{-} \rightarrow \mathrm{PEDOT}^{0}+M^{+} \mathrm{PSS}^{-}\right.$where $M^{+}$is a cationic impurity). ${ }^{44,45}$ This dedoping process causes a decrease in the conductivity $\sigma$ of the PEDOT:PSS film. EC transistors ${ }^{44-46}$ and bistable memories ${ }^{45}$ are examples operating in switching between the oxidized or reduced state of a conjugated polymer. Wang et al. ${ }^{47}$ visualized ion current in dedoping a fully oxidized polypyrrole film. According to their report, ions propagate with a "propagation front," which reflects the penetration of electric field inside the conducting polymer. Along the path which ions pass, polypyrrole film is reduced showing an apparent color change. Based on Wang et al.'s observation for the drift motion for ion propagation, the response rate of the PEDOT:PSS channel we experimentally obtained is related to the drift motion of ions. Microscopically, ion movement is through hopping with a preferential direction parallel to the electric field. Thus, the macroscopic result obtained $k\left(V_{G}\right)$ inside PEDOT:PSS using Eq. (2) can be rewritten as ${ }^{48} k$ $=k_{0} \exp \left[(e E R) /\left(2 k_{B} T\right)\right]$, where $E, R, e, k_{B}$, and $T$ are the electric field driving the ion, ion hopping distance, the ion charge, the Boltzman constant and temperature, respectively. $R$ is found as $R=\left(\frac{2 k_{B} T}{e E}\right)\left(\frac{V_{G}}{V_{0}}\right)$. Assuming the voltage drop along the ion penetration depth $d$ in $30 \mathrm{sec}$ is $\gamma V_{G}$ with electric field strength $E=\gamma V_{G} / d, \quad \gamma<1, R=\left(\frac{2 k_{B} T}{e \gamma}\right)\left(\frac{d}{V_{0}}\right) \approx 0.09 \frac{d}{\gamma}(\mathrm{nm})$ with $\gamma<1$ at room temperature for experimental value $V_{0}$ $=0.556(\mathrm{~V})$. In the case of $50 \%$ change in $I_{D}$ for $V_{G}=$ $+4.5 \mathrm{~V}$ in Fig. 4(a), using half of the thickness of the PEDOT:PSS film $d=40 \mathrm{~nm}$ yields $R>3.6 \mathrm{~nm}$. The obtained hopping distance corresponds to at least 3 repeat units of PEDOT:PSS away which is less probable for massive ions. A reasonable ion hopping distance $R<0.5 \mathrm{~nm}$ yields $d$ $<5.5 \gamma(\mathrm{nm})$ and $\gamma<1$. This result shows that ion penetrates 
less than one-tenth of the thickness of the PEDOT:PSS film. However, one-tenth reduction in the active channel thickness does not reflect $>20 \%$ change of $I_{D}$ for $V_{G}>+3.5 \mathrm{~V}$ in Fig. 4(a). Thus, the gradually dedoping (or depletion) picture is not appropriate to describe devices studied in this paper. The role of inserted mobile ions in devices studied is different from a typical electrochemical reaction.

Another evidence not supporting the electrochemical dedoping mechanism is the reduction of free carrier concentration $n$ of PEDOT:PSS. From $\sigma=n q \mu$, the change in $\sigma$ is dependent on the change in $n \mu$. A typical electrochemical (EC) reaction ${ }^{32}$ is understood as modulating $n$ mediated by a large amount of ions to change the doping level. Thus, $\sigma$ and $\mu$ respond accordingly. However, the conductance modulation of the active channel for devices studied shows a percolation phenomenon and we determined from $g\left(Q_{i o n}\right) / g_{0}$ that the approximately $2 \%$ hole charge on the PEDOT backbone being compensated by inserted ions is sufficient to suppress conductance up to three orders of magnitude. The $2 \%$ reduction in the free carrier concentration $\left(n \sim 10^{21} \mathrm{~cm}^{-3}\right)$ does not affect the metallic nature of the PEDOT:PSS. Instead of drift motion, mobile ions diffuse inside metallic PEDOT:PSS which is driven by the concentration gradient. The change of electrical potential in the PVP layer reflects the variation in the concentration gradient of inserted mobile ions in the active channel. To reach thermal equilibrium, the increase of chemical potential inside the active channel will reflect the increase of electrochemical potential in PVP. In the presence of mobile ions, $\sigma$ of the metallic polymer decreases dramatically but with only a few percentage decrease in $n$. The role of these inserted ions is to directly modulate $\mu$. The concentration of inserted ions determines $\mu$ of the system; hence $\sigma$. We note that the physical mechanism for this astonishing effect on conductivity modification through directly modulating $\mu$ has not been reported in the literature yet. Therefore, the ion diffusion coupled with the free carrier motion on the PEDOT backbone results in a new type of field effect mechanism.

$\mu_{F E T}$ and $R$ for the transistors studied do not give physical values in terms of a conventional FET and electrochemical transistor pictures. In addition, no significant modulation of $n$ is required to produce dramatic conductance suppression. Here, we propose a new model for metallic polymer transistors based on the unusual polymer morphology.

A doped polymer matrix contains an abundance of long polymer chains with their various small segments either parallel or nonparallel to each other resulting in a rich morphology. Generally, a doped polymer system can be viewed as ordered regions embedded in disordered regions. ${ }^{11,14,17,49}$ The size and the fraction of the ordered regions vary from system to system. Polymer chains are parallel to each other and well packed in the ordered regions while others are misoriented or coil-like or both in the disordered regions. The ordered regions are highly conductive and show the metallic properties while the disordered regions are poorly conductive and contain more free volume. This partially ordered morphological structure of doped polymers can be obtained experimentally from $x$-ray diffraction (XRD) measurements.

From XRD spectrum of a PEDOT:PSS free standing film taken in the range for $2 \theta=1^{\circ}-42^{\circ}$ [see Fig. 10(a)], we ob-
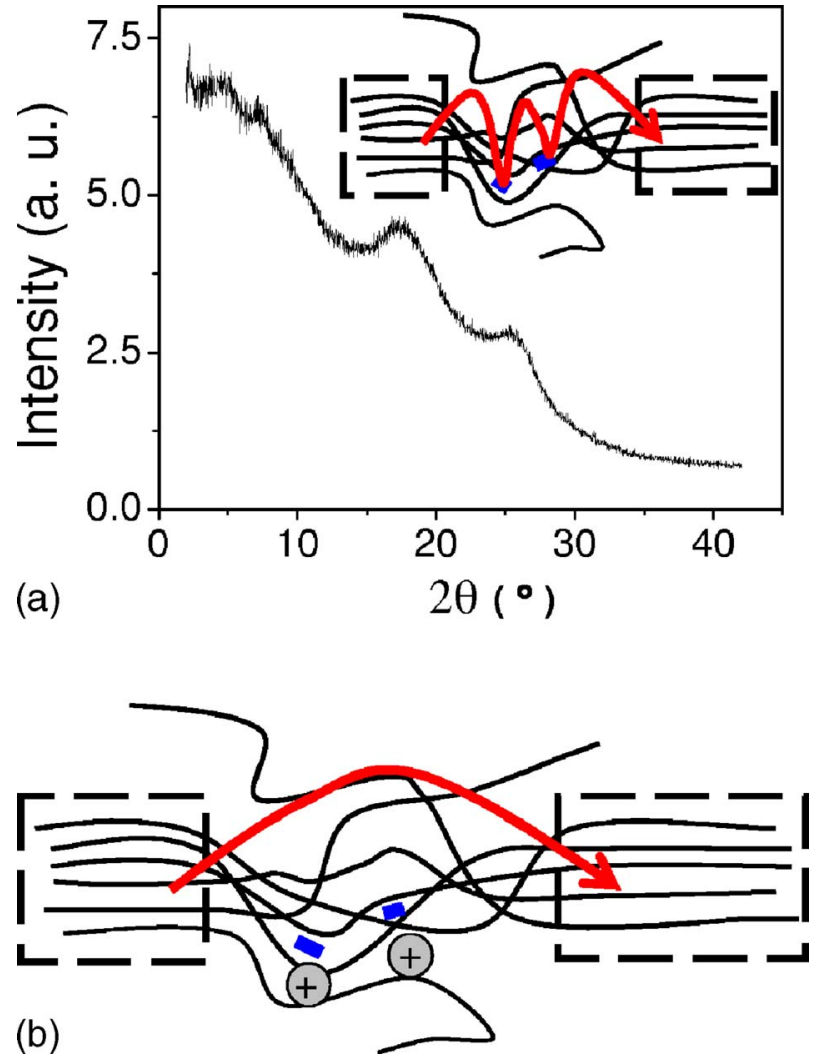

FIG. 10. (Color online). Intergrain hopping of charge carriers. (a) X-ray diffraction result for a free standing PEDOT:PSS film suggests a partially ordered morphological structure as in the schematic view shown in the inset. Carriers hop among localized states in disordered regions to conduct electricity. (b) The presence of inserted ions compensates localized states, thereby removing paths for easy hops between grains.

served intense broad bands centered at angular positions $2 \theta$ $\sim 25.5^{\circ}, 17^{\circ}, 7.5^{\circ}$, and $4.6^{\circ}$ which correspond to lattice plane spacing $d \sim 3.5 \AA, 5.2 \AA, 11.8 \AA$, and $18.8 \AA$, respectively. From literature reports for PEDOT doped with different dopants and stoichiometric, ${ }^{50,51}$ we assign the distance perpendicular to the thiophene plane, the size of three PEDOT rings and distance between thiophene chains on the same plane are $\sim 3.5 \AA, 11.8 \AA$, and $18.8 \AA$, respectively. A volume for a primitive lattice is estimated to be $\sim 800 \AA^{3}$. The estimated domain size for the ordered regions is approximately $21 \AA$ by using the Scherrer formula ${ }^{52}$ and the crystallinity of PEDOT:PSS is estimated to be $\sim 10 \%$. This XRD results confirm the inhomogeneous morphological structure of the PEDOT:PSS film.

Charge transport in the inhomogeneous morphological system is associated with charge hopping among ordered regions as shown in the inset in Fig. 10(a). Carriers are threedimensionally delocalized within the ordered regions but spatially localized in the disordered regions. Cross talk between ordered regions is accomplished by carriers hopping through localized charged states in the disordered regions. The random oriented nature of polymer chain in the disordered regions dominates the dc charge transport. Microscopically, electrons hop from a positively charged localized state 
to another positively charged state on the polymer backbone accompanying with polymer chain distortion due to dimerization. For each hop, the Coulomb repulsion energy compensates the local polymer chain deformation. Therefore, the spatial degree of freedom for charge to hop in disordered regions in polymer systems is determined by the electron wave function overlapping between nearby chains and of neighboring hopping sites on the same chain. For weak interchain electron wave function overlapping, carrier movement is more confined on a single polymer chain. Charge carriers mainly hop along the same polymer chain, but avoid the large energy barrier on the way by hopping to a neighboring chain resulting in a hopping dimension of $1+\varepsilon$, where $\varepsilon \ll 1$, quasi-1D VRH. The result of quasi-1D VRH charge transport mechanism of the PEDOT system suggests that intrachain hopping dominates the charge hopping process. Thus, the communication between polymer chains in disordered regions are relatively isolated in the PEDOT:PSS system.

Based on the transport on relatively isolated polymer chains in disordered regions, we studied the charge transport properties of the PEDOT:PSS system in the presence of inserted ions. We obtained from $g\left(Q_{i o n}^{\text {cell }}\right) / g_{0}$ a percolation phenomenon for conductance suppression with percolation threshold $Q_{C}^{\text {cell }} \approx 0.02$. According to the morphological structure of polymers, the inserted ions mostly distribute within porous disordered regions. We recalculated the percolation threshold $\left(Q_{C}^{\text {cell }}\right)^{\prime}$ by considering the fraction of disordered regions $(\sim 90 \%)$ and obtained $\left(Q_{C}^{\text {cell }}\right)^{\prime} \approx 0.02$. Approximately $2 \%$ reduction of charged states on the PEDOT backbone in the disordered regions through randomly neutralizing counterions by inserted cations can effectively alter the conductance of PEDOT:PSS up to three orders of magnitude. If we take $10 \%$ crystallinity in the PEDOT:PSS system and consider that the ordered regions as conductive cubes $(21 \AA$ for each side) homogeneously distributed in the media, the nearest conductive cubes are approximately $24.2 \AA$ apart, which corresponds with 2 hopping sites for a straight chain. Each conductive cube provides $\sim 14$ chains to randomly spread over the disordered regions to communicate with its neighboring cubes. There are approximately 10 hopping sites per chain neglecting any free volume in disordered regions. $\left(Q_{C}^{\text {cell }}\right)^{\prime} \approx 0.02$ yields at most 3 sites on those 14 chains being compensated by inserted ions can effectively suppress charge conduction between conducting regions. This also indicates that at most 3 polymer chains are responsible for intergrain communication if there is only one resonance hopping site on one chain. The astonished conduction modulation effect caused by a few percentage reduction of hopping states is attributed to the anisotropy charge hopping nature of a quasi-1D system. This small $\left(Q_{C}^{\text {cell }}\right)^{\prime}$ obtained here is also observed in other polymer blend systems. ${ }^{53,54}$

The percolation mechanism for conductance suppression through inserted ions is also supported by the resistivity measurement. The increase of $T_{0}$ with the increase of $\rho_{\alpha}$ [see Fig. 8(b)] reveals that the electric field effect in metallic polymer system is a bulk effect. The distribution of inserted ions happens throughout the whole bulk channel. The original conducting paths are either completely or partially cut after ions enter the active channel. The paths that contribute to the conductance after ion ingress have hopping sites partially removed. It should be pointed out again that this bulk effect is in contrast to the surface effect of the noted conventional field effect.

The partially cut polymer chains provide less hopping sites for carriers to hop through. This causes charge carriers to choose a different optimum condition for the next hop. An optimum hop ${ }^{38}$ is chosen to be a longer hopping distance for a lower energy difference site around the Fermi level. As a result, the corresponding energy $T_{0}\left\{=1 /\left[N\left(E_{F}\right) \xi\right]\right\}$ required to initiate a farther hop increases in the decrease of $N\left(E_{F}\right)$, assuming a constant $\xi$ for small $\left(Q_{C}^{\text {cell }}\right)^{\prime}$. The higher the $T_{0}$ is, the more the hopping sites are removed. Thus, the carrier hopping distance is longer in a large $\rho_{\alpha}$ state. Therefore, the hopping states remaining on a partially cut conducting path become more spatially localized [see Fig. 10(b)]. The probability for charge carriers to find a nearby hopping state strongly decreases. Consequently, $\mu$ decreases with the increase of carrier hopping distance.

We note here that we tried to examine this field effect behavior in other fully doped polymer films $(70-100 \mathrm{~nm}$ thick) such as polyaniline doped with camphorsulfonic acid (PAN:CSA) cast from $m$-cresol, modified electronic grade PEDOT:PSS (Aldrich) (ePEDOT:PSS) with the formula mentioned in the experimental section, and fully sulfonated polyaniline (NSPAN) cast from deionized water. The corresponding electrical conductivities are measured as $249 \mathrm{~S} / \mathrm{cm}, 0.1 \mathrm{~S} / \mathrm{cm}$, and $0.02 \mathrm{~S} / \mathrm{cm}$. We observed electricfield controlled conductance phenomena in metallic PAN:CSA and ePEDOT:PSS. The nonapparent electric field controlled phenomenon in low conductive NSPAN is presumably due to the comparable interchain and intrachain hopping probability in a relatively disordered system. ${ }^{55}$

We report a new type of working principle for conducting polymer based devices. The inserted ions diffuse to the poorly conducting disordered regions causing approximately $2 \%$ decrease in the free carrier concentration, i.e., $2 \%$ reduction in the hopping sites, but produce $10^{3}$ time suppression in conductance. This astonishing conductance modulation effect is achieved by directly modulating $\mu$ throughout the whole film. This new type of mechanism is also supported by optical measurements. ${ }^{56}$ According to the optical measurements, ${ }^{56}$ there was only a few percent decrease in the absorbance in the UV-visible rage but a dramatic decrease in the PEDOT:PSS conductance, with the substantial reduction of active channel conductance. Instead of UV-visible range, a substantial decrease in the infrared reflectance was observed which suggests that carriers are more localized after ions are inserted. More evidence supporting the proposed mechanism will be on a separate publication. ${ }^{57}$

We note that the conductance modulation in our PEDOT: PSS devices do not involve significantly opening a gap at the Fermi surface. This is the crucial difference from an electrochemical dedoping reaction by modulating charge carrier concentration through a large amount of ion charge flow. ${ }^{45,46}$ Though the device working principles are different, our devices show little difference in the $I_{D^{-}} V_{D}$ characteristics $^{44}$ and dynamic switching ${ }^{45}$ from an EC transistor. 


\section{CONCLUSION}

We have presented the working principle for metallic PEDOT based transistors based upon the unusual polymer morphology. The charge suppression mechanism in metallic PEDOT:PSS originates neither from gradually depleting of free charge carriers nor electrochemical dedoping reaction. The inserted ions in the PEDOT:PSS film randomly compensate $2 \%$ counterions in the porous disordered regions resulting in a dramatic reduction of charge conduction paths in the PEDOT system. The removal of hole states in disordered regions follows a percolation phenomenon with percolation threshold $\left(Q_{C}^{\text {cell }}\right)^{\prime} \approx 0.02$. This small percolation threshold is associated with the weak interchain charge transport mechanism of PEDOT. A small fraction removal of mediating hop- ping states near the Fermi level on charge transport paths causes carriers to hop over longer distance to conduct current leading to a conductor-nonconductor transition. This presented work proposes a new type of mechanism for the operation of metallic polymer based devices in contrast to the electrochemical doping-dedoping process, modulating free carrier concentration. This proposed mechanism should not be limited to PEDOT system, but common to other kinds of metallic polymer based three-terminal devices.

\section{ACKNOWLEDGMENTS}

This work was funded in part by DOE Grant No. DEFG02-01ER45931 and AFOSR Grant No. FA 9550-06-10175 .
${ }^{1}$ H. Sirringhaus, T. Kawase, R. H. Friend, T. Shimoda, M. Inbasekaran, W. Wu, and E. P. Woo, Science 290, 2123 (2000).

${ }^{2}$ N. T. Harrison, D. R. Baigent, I. D. W. Samuel, R. H. Friend, A. C. Grimsdale, S. C. Moratti, and A. B. Holmes, Phys. Rev. B 53, 15815 (1996).

${ }^{3}$ T. Kawase, H. Sirringhaus, R. H. Friend, and T. Shimoda, Adv. Mater. (Weinheim, Ger.) 13, 1601 (2001).

${ }^{4}$ J. A. Rogers, Z. Bao, A. Makhija, and P. Braun, Adv. Mater. (Weinheim, Ger.) 11, 741 (1999).

${ }^{5}$ Z. Bao, Y. Feng, A. Dodabalapur, V. R. Raju, and A. J. Lovinger, Chem. Mater. 9, 1299 (1997).

${ }^{6}$ J. C. Laquindanum, H. E. Katz, A. J. Lovinger, and A. Dodabalapur, Chem. Mater. 8, 2542 (1996).

${ }^{7}$ H. L. Cheng, W. Y. Chou, C. W. Kuo, F. C. Tang, and Y. W. Wang, Appl. Phys. Lett. 88, 161918 (2006).

${ }^{8}$ Y. - Y. Lin, D. J. Gundlach, S. F. Nelson, and T. N. Jackson, IEEE Electron Device Lett. 18, 606 (1997).

${ }^{9}$ Y. Jin, Z. Rang, M. I. Nathan, P. P. Ruden, C. R. Newman, and C. D. Frisbie, Appl. Phys. Lett. 85, 4406 (2004).

${ }^{10}$ C. Chiang, C. Chen, J. Kanicki, and K. Takechi, Appl. Phys. Lett. 72, 2874 (1998).

${ }^{11}$ J. P. Pouget, M. E. Jozefowicz, A. J. Epstein, X. Tang, and A. G. MacDiarmid, Macromolecules 24, 779 (1991); M. E. Jozefowicz, A. J. Epstein, J. P. Pouget, J. G. Masters, A. Ray, Y. Sun, X. Tang, and A. G. MacDiarmid, Synth. Met. 41, 723 (1991).

${ }^{12}$ R. S. Kohlman and A. J. Epstein, in Handbook of Conducting Polymers, 2nd ed., edited by T. A. Skotheim, R. L. Elsenbaumer, and J. R. Reynolds (Marcel Dekker, New York, 1998), p. 85.

${ }^{13}$ D. H. Kim, Y. D. Park, Y. Jang, H. Yang, Y. H. Kim, J. I. Han, D. G. Moon, S. Park, T. Chang, C. Chang, M. Joo, C. Y. Ryu, and K. Cho, Adv. Funct. Mater. 15, 77 (2005).

${ }^{14}$ R. S. Kohlman, J. Joo, Y. G. Min, A. G. MacDiarmid, and A. J. Epstein, Phys. Rev. Lett. 77, 2766 (1996).

${ }^{15}$ R. S. Kohlman, A. Zibold, D. B. Tanner, G. G. Ihas, T. Ishiguro, Y. G. Min, A. G. MacDiarmid, and A. J. Epstein, Phys. Rev. Lett. 78, 3915 (1997).

${ }^{16}$ J. Joo, S. M. Long, J. P. Pouget, E. J. Oh, A. G. MacDiarmid, and A. J. Epstein, Phys. Rev. B 57, 9567 (1998).

${ }^{17}$ V. N. Prigodin and A. J. Epstein, Physica B 338, 310 (2003).

${ }^{18}$ A. G. MacDiarmid, Angew. Chem., Int. Ed. 40, 2581 (2001).
${ }^{19}$ A. J. Epstein, F. C. Hsu, N. R. Chiou, and V. N. Prigodin, Curr. Appl. Phys. 2, 339 (2002).

${ }^{20}$ J. Lu, N. J. Pinto, and A. G. MacDiarmid, J. Appl. Phys. 92, 6033 (2002).

${ }^{21}$ H. Okuzaki, M. Ishihara, and S. Ashizawa, Synth. Met. 137, 947 (2003).

${ }^{22}$ N. R. Chiou, M.S. thesis, The Ohio State University, 2002.

${ }^{23}$ V. N. Prigodin, F. C. Hsu, Y. M. Kim, J. H. Park, O. Waldmann, and A. J. Epstein, Synth. Met. 153, 157 (2005).

${ }^{24}$ S. M. Sze, Semiconductor Devices (John Wiley, New York, 1985).

${ }^{25}$ W. W. Chiu, J. Travaš-Seejdić, R. P. Cooney, and G. A. Bowmaker, Synth. Met. 155, 80 (2005).

${ }^{26}$ A. G. MacDiarmid and A. J. Epstein, Synth. Met. 69, 85 (1995).

${ }^{27}$ J. Y. Kim, J. H. Jung, D. E. Lee, and J. Joo, Synth. Met. 126, 311 (2002).

${ }^{28}$ J. Quyang, Q. Xu, C. W. Chu, Y. Yang, G. Li, and J. Shinar, Polymer 45, 8443 (2004).

${ }^{29}$ These devices used NOA 65 optical adhesive purchased from Norland product Inc. as dielectric material and PEDOT:PSS as gate electrode.

${ }^{30}$ A. J. Epstein, J. H. Park, Y. M. Kim, F. C. Hsu, N. R. Chiou, O. Waldmann, and V. N. Pridogin, Polym. Prepr. (Am. Chem. Soc. Div. Polym. Chem.) 45, 149 (2004).

${ }^{31}$ B. N. Streetman and S. Banerjee, Solid State Electronic Devices (Prentice Hall, New Jersey, 1990).

${ }^{32}$ A. J. Bard and L. R. Faulkner, Electrochemical Methods: Fundamentals and Applications (John Wiley, New York, 2001).

${ }^{33}$ For silicon MOSFETs we made, we measured the leakage current is less than $10 \mathrm{pA}\left(\sim 10^{-12} \mathrm{~A} / \mathrm{mm}^{2}\right)$.

${ }^{34}$ The volume for a PEDOT cell is estimated to be $\sim 800 \AA^{3}$ from the XRD measurement presented in the discussion section.

${ }^{35} \mathrm{H}$. Böttger and V. V. Bryksin, Hopping Conduction in Solids (VCH, Deerfield Beach, FL, 1985), p. 123.

${ }^{36}$ F. C. Hsu et al. (unpublished).

${ }^{37}$ F. C. Hsu, Ph.D. thesis, The Ohio State University, 2005.

${ }^{38}$ N. F. Mott and M. Kaveh, Adv. Phys. 34, 329 (1985).

${ }^{39}$ J. Y. Kim, J. H. Jung, D. E. Lee, and J. Joo, Synth. Met. 126, 311 (2002).

${ }^{40}$ H. G. O. Sandberg, T. G. Bäcklund, R. Österbacka, and H. Stubb, Adv. Mater. (Weinheim, Ger.) 16, 1112 (2004). 
${ }^{41}$ T. G. Bäcklund, H. G. O. Sandberg, R. Österbacka, and H. Stubb, Appl. Phys. Lett. 85, 3887 (2004).

${ }^{42}$ T. F. Otero and I. Boyano, J. Phys. Chem. B 107, 6730 (2003).

${ }^{43} \mathrm{O}$. Walddman et al. (unpublished).

${ }^{44}$ D. Nilsson, N. Robinson, M. Berggren, and R. Forchheimer, Adv. Mater. (Weinheim, Ger.) 17, 353 (2005).

${ }^{45}$ D. Nilsson, M. Chen, T. Kugler, T. Remonen, M. Armgarth, and M. Berggren, Adv. Mater. (Weinheim, Ger.) 14, 51 (2002).

${ }^{46}$ J. W. Thackeray, H. S. White, and M. S. Wrighten, J. Phys. Chem. 89, 5133 (1985).

${ }^{47}$ X. Wang, B. Shapiro, and E. Smela, Adv. Mater. (Weinheim, Ger.) 16, 1605 (2002).

${ }^{48}$ N. F. Mott and R. W. Gurney, Electronic Processes in Ionic Crystal, 2nd ed. (Dover, New York, 1948).

${ }^{49}$ V. N. Prigodin and A. J. Epsten, Synth. Met. 125, 43 (2002).

${ }^{50}$ L. Niu, C. Kvarnstrőm, K. Frőberg, and A. Ivaska, Synth. Met.
122, 425 (2001).

${ }^{51}$ K. E. Aasmundtveit, E. J. Samuelsen, L. A. A. Pettersson, O. Inganäs, T. Johansson, and R. Feidenhans'l, Synth. Met. 101, 561 (1999).

${ }^{52}$ A. Guiner, Theorie et Technique de la Radio-crystallographie (Dunod, Paris, 1950).

${ }^{53}$ G. Du, J. Avlyanov, C. Y. Wu, K. G. Reimer, A. G. MacDiarmid, and A. J. Epstein, Synth. Met. 85, 1339 (1997).

${ }^{54}$ O. T. Ikkala, J. Laakso, K. Väkiparta, E. Virtanen, H. Ruohonen, H. Järvinen, T. Taka, P. Passiniemi, J.-E. Österholm, Y. Cao, A. Anfreatta, P. Smith, and A. J. Heeger, Synth. Met. 69, 97 (1995).

${ }^{55}$ F. C. Hsu and A. J. Epstein (unpublished).

${ }^{56}$ Y. M. Kim, Ph.D. thesis, The Ohio State University, 2005.

${ }^{57}$ F. C. Hsu and A. J. Epstein (unpublished). 